Research Article

\title{
Simulation of Interference Effects of UWB Pulse Signal to the GPS Receiver
}

\author{
Xin Huang $\mathbb{D}$, Yazhou Chen $\mathbb{D}$, and Yuming Wang $(\mathbb{D}$ \\ Army Engineering University, Shijiazhuang Campus, National Key Laboratory on Electromagnetic Environment Effects, \\ Shijiazhuang 050000, China
}

Correspondence should be addressed to Yazhou Chen; chen_yazhou@sina.com

Received 27 March 2021; Accepted 10 July 2021; Published 19 July 2021

Academic Editor: Shiping Wen

Copyright (c) 2021 Xin Huang et al. This is an open access article distributed under the Creative Commons Attribution License, which permits unrestricted use, distribution, and reproduction in any medium, provided the original work is properly cited.

Ultra-wideband (UWB) pulse signal has an extremely narrow pulse width and wide frequency bandwidth, which overlaps with the operating frequency band of Global Position System (GPS) receivers, posing a potential threat to their performance. In response to this problem, through mathematical analysis and software simulation, the effects of UWB pulse signal under time-hopping-pulse position modulation (TH-PPM) on the performance of GPS receivers were studied. First, the expression and waveform of the UWB pulse signal were analyzed in the time-frequency domain, and it is concluded that the pulse repetition frequency (PRF) mainly affects the discrete spectrum of the UWB signal and the TH code period mainly affects the continuous spectrum. On this basis, the simulation on the power spectral density (PSD) of GPS signal and UWB signal under different pulse parameters was represented, from which a conclusion can be drawn that the PRF is the main factor impacting the PSD of the GPS signal. Furthermore, this paper analyzed the degradation of GPS receiver equivalent carrier-to-noise ratio $\left(C / N_{0}\right)$ and $\mathrm{C} / \mathrm{A}$ code demodulation bit error rate (BER) under UWB interference, which are the crucial evaluating indicators of GPS signal quality. Eventually, we theoretically calculated the minimum interference level of the UWB interference signal to the GPS receiver, providing a theoretical reference for reducing the interference effects of UWB pulse signal on the performance of GPS receivers.

\section{Introduction}

In the 1960s, the problem of ultra-wideband radio frequency (UWB RF) signal generation was solved with the numerous efforts of many researchers [1]; with the rapid development of electronic communication technology, the UWB system is gradually used widely in all sorts of fields such as weapons and equipment, navigation, and radar imaging $[2,3]$. The UWB system is a major revolution in traditional communication methods, which is different from conventional communication methods based on sinusoidal carrier modulation. UWB uses nanosecond-level extremely narrow pulses as information carriers, having high information transmission rate, high processing gain, and strong penetration, which was officially accepted for entering commercial applications by the Federal Communications Commission (FCC) in 2002. In consideration of the broad application of UWB in wireless communication, it is a serious issue to avoid spectrum conflicts between UWB systems and other narrowband systems and suppress the UWB pulse signal interference. Therefore, the FCC has strictly regulated the spectrum range and transmit power of UWB systems. However, existing studies have shown that the ultra-wide spectrum characteristics of UWB pulse signals will still cause degradation of the functions of existing frequency-using systems, especially for GPS signals whose power is much lower than the noise floor, consequently extremely vulnerable to the RF interference [4-7].

GPS is a satellite navigation system developed by the United States Department of Defense to provide accurate positioning, accurate speed measurement, and high-precision standard time. Accurate, continuous, and high-quality GPS signals are the key to effective positioning and navigation on the ground. However, GPS satellites are located in a circular orbit around $20,200 \mathrm{~km}$ above Earth's surface. After their satellite signals propagate through space, the 
signal strength is already very weak when they reach Earth's surface. The jammer with interference power of $1 \mathrm{~W}$ performs noise interference on the GPS receiver, which can make the GPS receiver within a range of $22 \mathrm{~km}$ lose its normal working ability, showing that the GPS signal has an inherent vulnerability. The UWB signal has a very wide spectrum and coverage, whose spectrum energy can easily fall into the GPS receiver and cause interference to the normal operation of the device. Therefore, the study of UWB system interference to GPS has important practical significance. Literature [8] derived accurate closed-form analytical expressions of receiver output and the postcorrelation carrier-to-noise of GPS signal, which provided the theoretical basis to analyze the pulse interference effects. Through numerical calculation and experimental research, literature [9] obtained that when the UWB pulse repetition frequency falls in the working bandwidth of the navigation receiver, the pulse amplitude and repetition frequency are inversely proportional when the same interference effect is achieved, and the product of them is a constant. Based on the PSD analysis of the UWB interference signal and BDS signal, the simulation of literature [10] showed that the octal PAM UWB signal is about $13 \mathrm{~dB}$ higher than the UWB radar signal interference power under the same parameters. In addition, the research analyzed the influence of the UWB signal on the intermediate frequency output of receivers under different systems and different powers.

Aiming at the problem of UWB pulse signal interference to GPS signals, this paper establishes a UWB signal model under typical TH-PPM modulation. From the signal characteristics, we analyze the influence of different PRFs and TH code periods on the PSD of UWB signals. On this basis, combining the relationship between the pulse repetition frequency and the bandwidth of GPS spread spectrum system, the equivalent $C / N_{0}$ and BER degradation of the receiver are derived with the increase of UWB signal power, meanwhile calculating the minimum interference level when the UWB signal leads to the tracking loss of the GPS receiver. In the end, effective measures are proposed to provide a reference for suppressing the interference of the UWB pulse signal with the GPS signal.

\section{Model of the TH-PPM UWB System}

2.1. Theoretical Analysis. The FCC defines UWB as a signal with an absolute bandwidth greater than $500 \mathrm{MHz}$ or a relative bandwidth (the ratio of the signal spectrum bandwidth to its center frequency) greater than or equal to $25 \%$, whose signal frequency range is $3.1-10.6 \mathrm{GHz}$, and the effective isotropic transmit power should not be higher than $-41.3 \mathrm{dBm} / \mathrm{MHz}[11]$.

The common way to generate the UWB signal is the impulse radio (IR), which transmits pulse signals in a very short time, and the resulting pulses have the characteristics of narrow pulse width and stable waveform. This paper adopts the TH- PPM UWB signal, which can effectively avoid communication interruption and data repeated transmission in multiuser situations. The pulse expression in the time domain can be written as

$$
i(t)=\sum_{j=-\infty}^{+\infty} g\left(t-j T_{f}-C_{j} \cdot T_{c}-a_{j} \varepsilon\right),
$$

where $g(t)$ represents the transmitted pulse waveform. Considering the actual engineering application, the waveform often uses the second derivative function of the Gaussian pulse, which can be written as

$$
g(t)=\frac{t^{2}-\sigma^{2}}{\sqrt{2 \pi} \sigma^{5}} \cdot e^{\left(-t^{2} / 2 \sigma^{2}\right)} .
$$

$T_{f}$ is the average pulse repetition period before modulation; $T_{c}$ is the additional time shift of each pulse of the time-hopping code; $C_{j}$ is the time-hopping code sequence; $\alpha_{j}$ is the binary information sequence; and $\alpha_{j} \varepsilon$ is the pulse displacement caused by the PPM modulation.

A typical time-domain waveform of the TH-PPM UWB pulse signal is shown in Figure 1. The pulse position of the modulated UWB signal is determined by the TH code pseudorandom sequence, which is translated randomly within a chip, and the randomized UWB signal greatly tends to be white noise in spectral characteristics.

From equation (1), it can be derived that when the probability of 0 and 1 of $\alpha_{j}$ appears, the power spectral density of the TH-PPM UWB signal can be expressed as

$$
\begin{aligned}
I(f)= & \frac{1}{2 T_{f}}|P(f)|^{2}\left(1-\cos ^{2}((\pi f \delta)) \sin c^{2}\left(\pi f N_{p} T_{c}\right)\right) \\
& +\frac{1}{2 T_{f}^{2}} \sum_{k=-\infty}^{\infty}\left|P\left(\frac{k}{T_{f}}\right)\right|^{2} \cos ^{2}(\pi f \delta) \sin c^{2}\left(\pi f N_{p} T_{c}\right) \delta\left(\frac{f-k}{T_{f}}\right) .
\end{aligned}
$$

$P(f)=\int_{-\infty}^{\infty} g(t) e^{-i 2 \pi f t} \mathrm{~d} t$ and $N_{p}$ is the pseudorandom sequence (TH code) period of the TH-PPM UWB signal. It is shown from equation (3) that the PSD of the TH-PPM UWB signal consists of a continuous part and discrete spectral lines whose frequencies are integer multiples of the PRF. $I_{c}(f)=\left(1 / 2 T_{f}\right)|P(f)|^{2}\left(1-\cos ^{2}(\pi f \delta) \sin c^{2}\left(\pi f N_{p} T_{c}\right)\right)$ which is the continuous spectrum part, and its envelope obeys a cosine envelope with a period of $1 / \delta$. $I_{d}(f)=$

$\left(1 / 2 T_{f}^{2}\right) \sum_{k=-\infty}^{\infty}\left|P\left(k / T_{f}\right)\right|^{2} \cos ^{2}(\pi f \delta) \sin c^{2}\left(\pi f N_{p} T_{c}\right) \delta(f-$ $k / T_{f}$ ) which is the discrete spectrum part. It is not difficult to deduce that PRF and the TH code period $N p$ have a significant influence on the PSD of UWB signals.

2.2. Simulation of UWB PSD. Next, we use software to simulate the spectral characteristics of TH-PPM UWB signals under different PRF and Np. Figure 2 shows the PSD of UWB signal under the same $N p$ and different PRF, and Figure 3 shows the UWB signal PSD under the same PRF and different $N p$.

In Figure 2, it is shown that PRF mainly affects the sparseness and pulse amplitude of the discrete spectral line part of the UWB PSD. With the PRF increasing, the discrete spectral line interval increases and the number of strong peak spectral lines decreases, resulting in a decrease in the average power of the UWB interference signal in a certain 


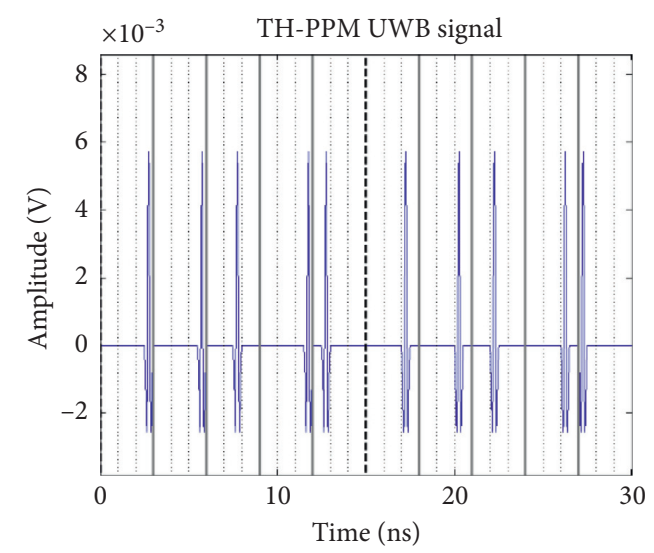

FIgURE 1: Time-domain waveform of UWB pulse signal.

frequency band, eventually weakening the interference effect; at the same time, the strongest spectral line power at the peak frequency of $2 \mathrm{GHz}$ is about $-106 \mathrm{dBm}$, and it does not change with the increase of repetition frequency.

In Figure 3, the change of $N p$ almost does not affect the position and amplitude of the strong peak discrete spectral line, while mainly affecting the envelope of the continuous spectral line. As the period increases, the peak of the continuous spectrum decreases, and the number of envelopes decreases and tends to be flat, which results in the weakening of the interference effect; after increasing to a certain value, the degree of spectral line changes slows down. It can be predicted that, with the continuous increase of $N p$, the UWB pulse position and the discrete spectral line interval also tend to be randomized, so that the power spectral density of the continuous spectral line part in the UWB signal has the feature of band-limited white noise. The performance in the frequency domain can be approximated as broadband Gaussian white noise.

\section{Analysis of UWB Pulse Signal Interference to the GPS Signal}

3.1. GPS Signal Structure. The GPS satellite signal structure includes three signal levels: navigation message, pseudocode, and carrier. Firstly, the pseudocode is used to spread spectrum modulation of the navigation message, the code rate is expanded from $50 \mathrm{bps}$ to $1.023 \mathrm{Mcps}$, and then the combined code is BPSK modulated on the carrier to form the final navigation signal to be broadcast by the satellite [12]. Taking the L1 carrier modulated by the C/A code as an example, the signal received by the $j_{-}{ }^{\text {th }}$ GPS satellite can be written as

$$
s^{(j)}(t)=A D^{(j)}\left(t-\tau^{j}\right) C^{(j)}\left(t-\tau^{j}\right) \cos \left(2 \pi\left(f_{1}+f_{d}^{j}\right) t+\varphi^{j}\right),
$$

where $A$ is the amplitude of the navigation signal; $D(t)$ is the navigation data code; $C(t)$ is the code of $\mathrm{C} / \mathrm{A} ; f_{1}$ is the center frequency of the L1 carrier; $f_{d}$ is the Doppler frequency shift of the satellite signal; $\tau$ is the signal transmission delay from the transmitter to the receiver; and $\varphi$ is the initial phase of the received carrier. The power spectral line of the codecarrying navigation information after being modulated by the C/A code is shown in Figure 4.

As shown in Figure 4, the C/A code of the GPS signal is a discrete spectral line that conforms to the sinc function envelope. In addition, the landing level of the GPS signal is very weak, and it is very susceptible to interference from the surrounding electromagnetic environment. Once the receiver-related processor leaks a strong pulse in the UWB signal which is mixed into the GPS signal, it is bound to pose a great threat to the performance of the receiver.

\subsection{Influence of Key Parameters of TH-PPM UWB Pulse} Signal on the PSD of GPS Signal. In order to study how the TH-PPM UWB system affects GPS signals, the following parameters are adopted for the simulation analysis: UWB pulse signal width is $0.5 \mathrm{~ns}$, the amplitude of the secondorder Gaussian pulse is $1 \mathrm{~V}$, the time shift introduced by PPM is $0.5 \mathrm{~ns}$, and $N p$ is 10 . We choose three different groups of PRF: PRF1 $=10 \mathrm{MHz}, \quad \mathrm{PRF} 2=15.75 \mathrm{MHz}$, PRF3 $=20 \mathrm{MHz}$, and GPS L1 carrier center frequency $f_{0}=1575.42 \mathrm{MHz}$. Within the $20 \mathrm{MHz}$ bandwidth of the L1 carrier center frequency, the frequency domain relationship of three UWB signal spectral lines under different PRFs is fitted, as shown in Figure 5.

It is graphically shown from Figure 5 that when $\mathrm{PRF}=15.75 \mathrm{MHz}$, its integer multiples happen to fall at the GPS carrier center frequency, and there is an obvious spectral energy spike that coincides with the GPS L1 main lobes, which is easy to mix into the acquisition and tracking process through the receiver front-end filter, effectively reducing the satellite signal $C / N_{0}$; when $P R F 1=10 \mathrm{MHz}$, the amplitude of the discrete spectral lines is generally low. Although there is a pulse spike near the center frequency, the signal power is low, and the interference to the satellite signal is weak; when PRF3 $=20 \mathrm{MHz}$, the peak value of the spectral energy is larger than the $10 \mathrm{M}$ repetition frequency, but the peak position has a large deviation from the center frequency.

It can be predicted that when the integral multiple of the pulse repetition frequency does not fall in the GPS working bandwidth and there is no single pulse with strong amplitude near the center frequency, the UWB interference effect is weak; when the integral multiple of the pulse repetition frequency just falls in the center frequency of the GPS signal, the interference effect is the most obvious, and it has the greatest impact on GPS signals. Within the working bandwidth of the GPS receiver, the smaller the pulse repetition frequency of the UWB signal, the smaller the average power of the signal, and the lower the degree of interference to the GPS signal.

In order to further explore the impact of the UWB signal when the PRF integer multiples fall in the working bandwidth of the GPS signal while keeping other parameters the same, the UWB PRF was simulated at $7.875 \mathrm{MHz}$, $15.75 \mathrm{MHz}, 31.5 \mathrm{MHz}$, and 78.75 MHz. The PSD of the UWB signal in the GPS operating frequency band is shown in Figure 6 . 


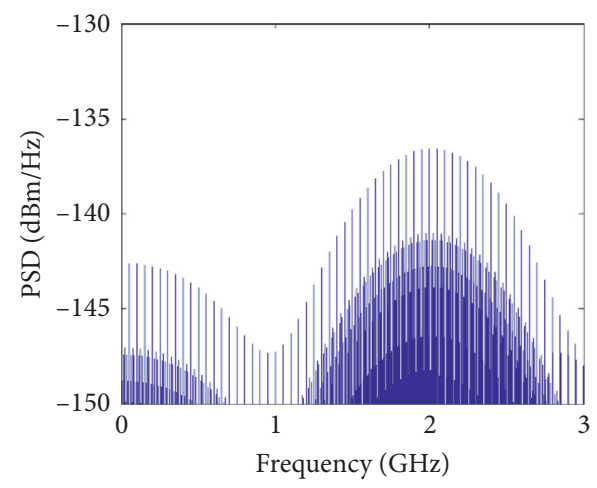

(a)

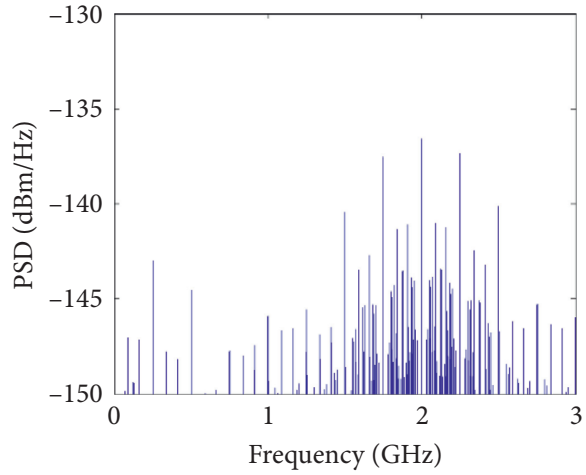

(b)

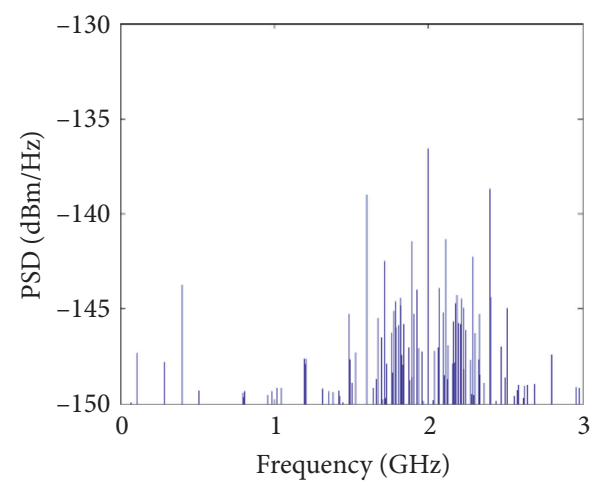

(c)

Figure 2: The PSD of TH-PPM UWB $(N p=10)$. (a) PRF $=10 \mathrm{MHz}, N p=10$. (b) PRF $=50 \mathrm{MHz}, N p=10 .(\mathrm{c}) \mathrm{PRF}=80 \mathrm{MHz}, N p=10$.

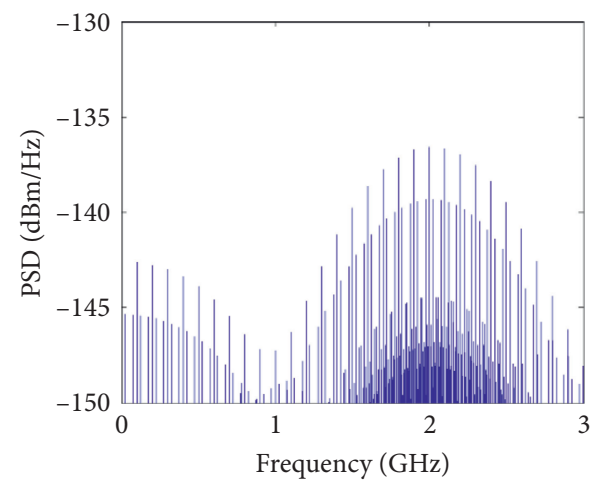

(a)

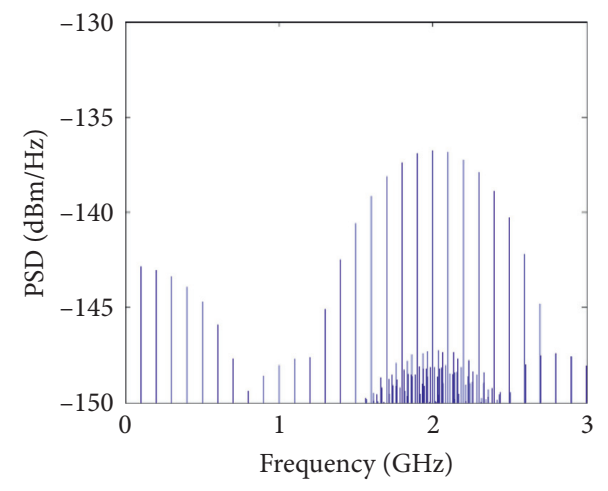

(b)

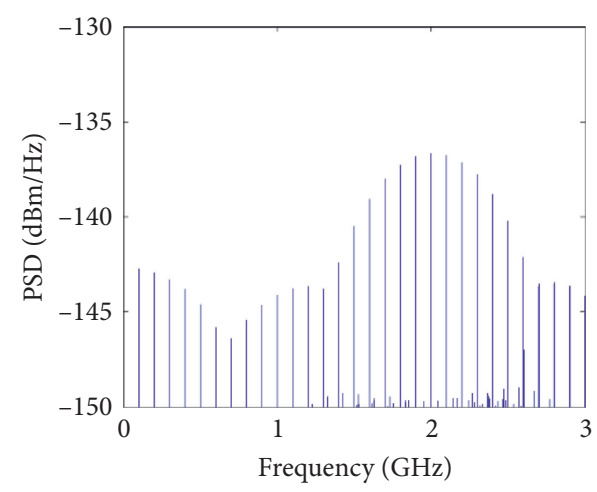

(c)

Figure 3: The PSD of TH-PPM UWB (PRF = 20 MHz). (a) PRF=20 MHz, $N p=10$. (b) PRF =20 MHz, $N p=50 .(\mathrm{c}) \mathrm{PRF}=20 \mathrm{MHz}, N p=100$. 


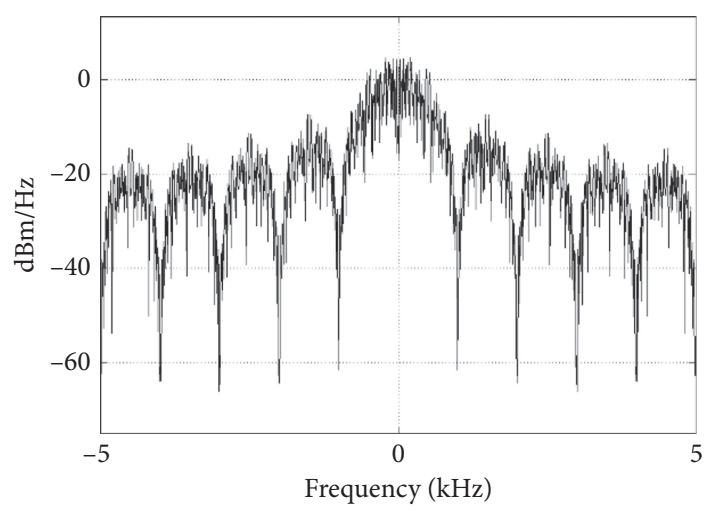

FIgURE 4: Power spectral of spread spectrum signal.

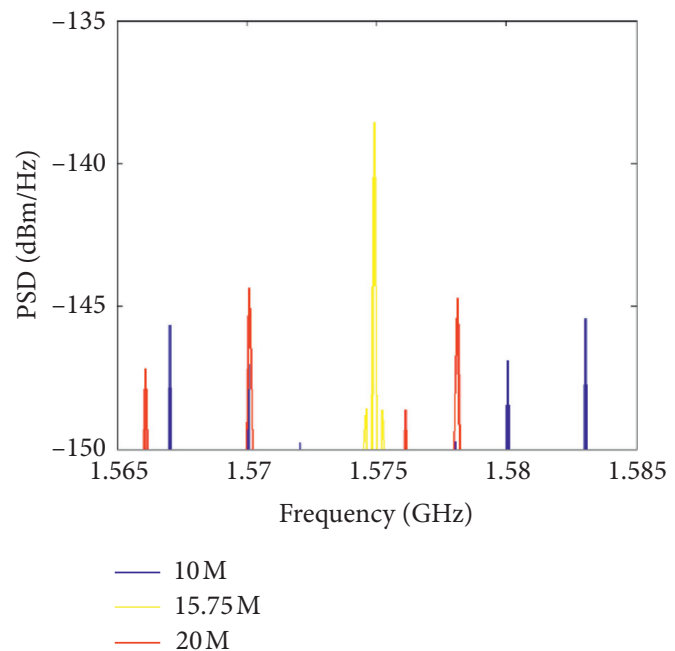

FIgURE 5: Comparison of power spectral lines of UWB signals under three different conditions.

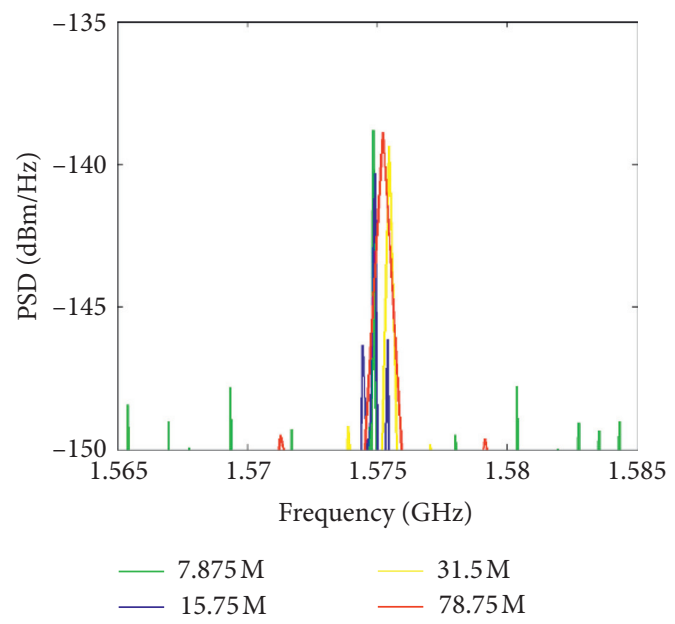

Figure 6: Power spectral density of UWB signal.

In Figure 6, the four different integer multiples of PRF all fall at the center frequency of the GPS carrier, which constitutes a large interference to the main peak of the satellite signal. With the increase of PRF, the UWB single pulse width at the center frequency of the GPS increases. The interference signal energy is more concentrated, and the interference effect is enhanced.

Considering the influence of the $\mathrm{TH}$ code period, the repetition frequency of $15.75 \mathrm{MHz}$ is selected, and the PSD of UWB pulse signal at the center frequency of the L1 carrier under three different $N p$ 's of 10, 30, and 50 can be obtained by simulation, as shown in Figure 7 .

It can be seen from Figure 7 that the power of the UWB signal at the L1 intermediate frequency under three different cycles is $-138.6 \mathrm{dBm},-139.2 \mathrm{dBm},-140.3 \mathrm{dBm}$, respectively. Although the power of the interference signal gradually decreases with the increase of $N p$, the change is not obvious, and the change has little effect on the interference to the GPS signal.

\section{Impact of the UWB Pulse Signal on the GPS Receiver Performance}

The GPS receiver uses a series of code correlation operations to calculate the pseudorange, carrier phase, Doppler shift, and other satellite navigation measurements from the satellite signal. The equivalent $C / N_{0}$ and $\mathrm{C} / \mathrm{A}$ code demodulation BER are two important aspects to measure its performance and positioning accuracy.

4.1. Equivalent $C / N_{0}$. In the absence of external interference, the satellite signal is downconverted by the RF front-end and the signal $C / N_{0}$ entering the correlator can be written as

$$
\frac{C}{N_{0}}=P_{R}-N_{0}-L_{f e}
$$

where $N_{0}=k T$ is the power spectral density of system thermal noise, $L_{f e}$ is the loss of the RF front end of the GPS receiver, and $P_{R}$ is the GPS signal power. Taking $T=290 \mathrm{~K}$, $P_{R}=-160 \mathrm{dBW}, L_{f e}=4 \mathrm{~dB}$ and substituting into equation (5), the initial $C / N_{0}$ of GPS under the lowest received power condition is about $40 \mathrm{~dB} \mathrm{~Hz}$. When GPS satellite signals suffer from external radio frequency interference and the $\mathrm{Cl}$ $N_{0}$ drops below the tracking threshold of the receiver, the receiver will not be able to capture and track satellite signals, thus losing normal navigation and positioning functions. Without considering environmental noise and other electromagnetic interference and only UWB signal interference, the equivalent $C / N_{0}$ is

$$
\left[\frac{C}{N_{0}}\right]_{e q}=\frac{1}{\left(1 /\left(C / N_{0}\right)\right)+((J / S) / Q R)},
$$

among them, $J / S$ is the ratio of interference signal power to useful signal power,

$$
\frac{J}{S}=10 \log \left[Q R\left[\frac{1}{{ }_{10}\left(\left[C / N_{0}\right]_{e q} / 10\right)}-\frac{1}{10\left(\left(C / N_{0}\right) / 10\right)}\right]\right](\mathrm{dB}) \text {. }
$$

$Q$ is the anti-interference quality factor, which characterizes the ability of the GPS system to resist different types 


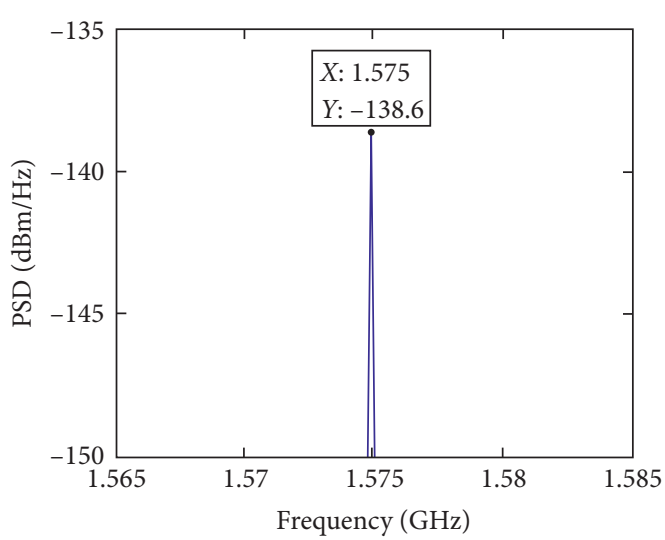

(a)

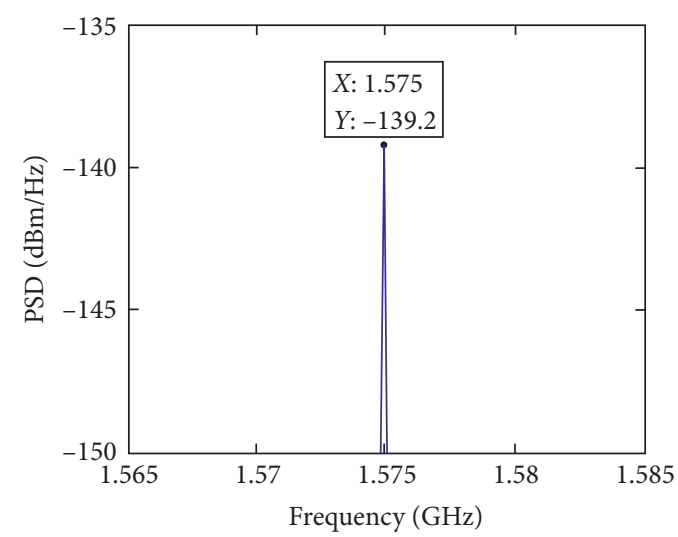

(b)

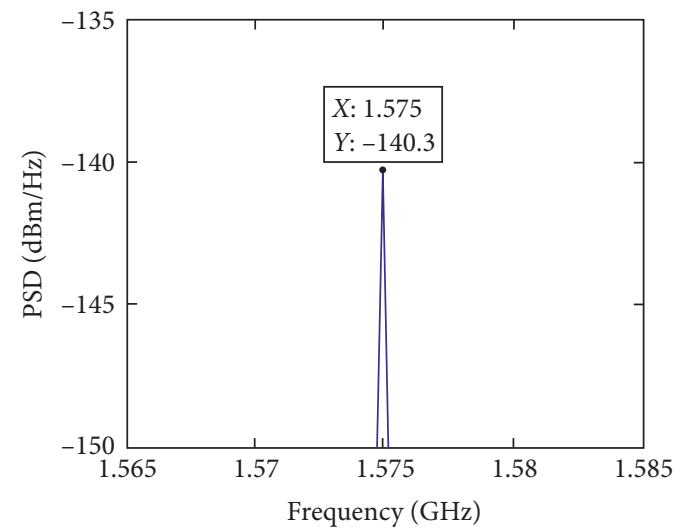

(c)

Figure 7: UWB signal power spectrum at PRF $=15.75 \mathrm{MHz}$. (a) $N p=10$. (b) $N p=30$. (c) $N p=50$.

of interference, and $R$ is the code rate of the $\mathrm{C} / \mathrm{A}$ code (1.023 Mcps). The greater the UWB interference signal power, the smaller the equivalent $C / N_{0}$ ratio of the GPS receiver, and the more energy of the interference signal falling into the receiver's correlator, causing the receiver to lose the symbols and carriers of the satellite signal at any time. According to literature [13], the satellite tracking threshold of a certain type of navigation receiver is $32 \mathrm{~dB} \mathrm{~Hz}$. With this receiver as the research object, the equivalent $C / N_{0}$ of the GPS receiver under UWB interference can be obtained as shown in Figure 8.

When $\left[C / N_{0}\right]_{e q}$ is higher than the tracking threshold, the real-time $C / N_{0}$ changes nonlinearly with the power of the interference signal and decreases slowly; when it falls below the tracking threshold, the receiver acquisition and tracking has been completely lost and cannot obtain the accurate satellite information. At this time, the equivalent $C / N_{0}$ and UWB interference signal power are approximately linearly distributed. When the receiver reaches the tracking threshold under the GPS L1 carrier, the interference signal ratio $J / S$ is $30 \mathrm{~dB}$ from equation (7). The power ratio of the UWB interference signal to the satellite signal is $J / S=J s-P_{R}$, where $P_{R}$ is the power $(-160 \mathrm{dBW})$ of GPS received signal, and the lowest interference level $J s=1 \times 10^{-13} \mathrm{~W}(-130 \mathrm{dBW})$ can be calculated. When the interference signal of UWB pulse enters the receiver with a power higher than

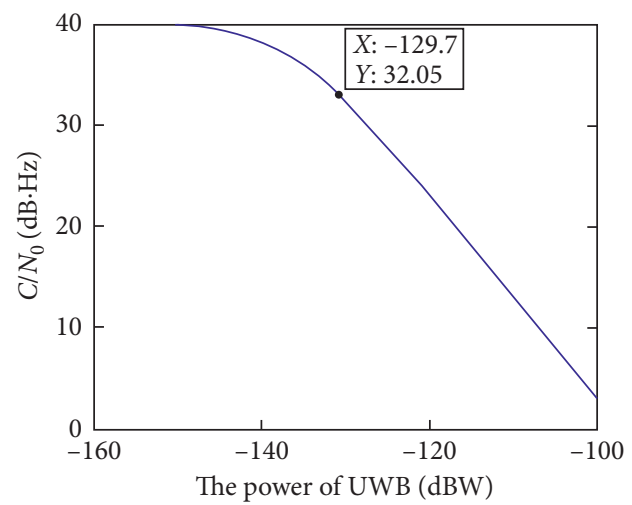

FIgUre 8: Receiver $\left[C / N_{0}\right]_{e q}$ under UWB interference.

$-130 \mathrm{dBW}$, it will interfere with the loop tracking process to a large extent, causing loss of positioning.

4.2. Demodulation BER. After the data code in the GPS signal is modulated by the spread spectrum of the pseudorandom sequence, the frequency band is greatly expanded, and the signal system presents the characteristics of a spread spectrum system. Under the UWB signal interference, the useful signal that leaks through the radio frequency filter of the receiver is mixed with some interference 
signals, which may cause the binary bit of the C/A code to be inverted during the BPSK demodulation, which reduces the demodulation accuracy.

The following will be discussed based on the relationship between the PRF of the UWB signal and the spread spectrum system bandwidth $B_{d}, k=\mathrm{PRF} / B_{d}$, which is the ratio between the UWB pulse repetition frequency and the bandwidth of the GPS system.

When PRF is greater than $B_{d}(k>1)$, there is essentially no discrete spectrum in the power spectrum of the interference signal output by the GPS receiver. The UWB interference signal can be approximated by the Gaussian model of white noise, which is equivalent to increasing the noise floor of the GPS receiver. Then, the demodulation BER of the receiver is

$$
P_{e}=Q\left[\sqrt{\frac{2 E_{b}}{N_{0}+P_{u}}}\right] .
$$

When PRF is less than $B_{d}(k<1)$, there will be at least one discrete spectral line of UWB signal falling within the GPS signal bandwidth. At this time, the interference mainly comes from the discrete part, which is equivalent to pulse interference. Then, the demodulation BER of the receiver is

$$
P_{e}=k Q\left[\sqrt{\frac{2 E_{b}}{N_{0}+\left(P_{u} / k\right)}}\right]+(1-k) Q\left[\sqrt{\frac{2 E_{b}}{N_{0}}}\right] .
$$

In the formula above, $E_{b}$ is the bit energy of the GPS signal, $N_{0}$ is the unilateral PSD of white noise, and $P_{u}$ is the PSD of UWB interference signal. The equivalent $C / N_{0}$ is $\left[C / N_{0}\right]_{e q}=\left(E_{b} / N_{0}\right) \times\left(R / B_{d}\right)$, by combining equations (6), (8), and (9). It can be shown that the PSD of UWB interference signals in the GPS working frequency band will greatly affect the equivalent $C / N_{0}$ of the receiver, which will lead to an increase in the system BER. The demodulation performance decreases and ultimately affects the ranging accuracy of the GPS receiver. Figure 9 shows the performance curve of GPS demodulation BER with $J / S$ variation under the interference of Gaussian white noise. When $J / S$ is less than $-20 \mathrm{~dB}$, the system BER is similar to the BER in the noninterference state, and the interference effect is weak; with the continuous increase of $J / S$, the BER gradually increases, and the BER approaches 0.5 when $J / S$ is at about $30 \mathrm{~dB}$. At this time, the system performance is seriously degraded, and the navigation message data cannot be demodulated correctly, which is consistent with the result in Section 3.1. In addition, with the increase of $E_{b} / N_{0}$, the demodulation error performance of GPS is getting better and better.

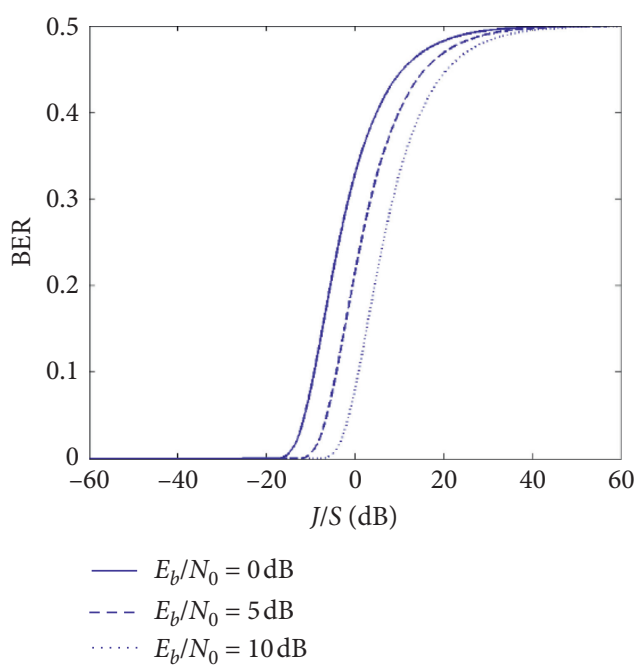

Figure 9: Bit error rate with a variation of $J / B$.

\section{Conclusions}

This paper studies the interference mechanism of the THPPM UWB pulse signal to the GPS receiver. Through theoretical analysis, power calculation, and software simulation, the following conclusions can be drawn:

(1) The pulse repetition frequency of TH-PPM UWB pulse signal mainly affects discrete spectral lines of spectral density. The greater the repetition frequency, the greater the spectral line spacing, and the smaller the pulse amplitude, yet it has little effect on the peak frequency; the $\mathrm{TH}$ code period mainly affects the continuous spectral line, and the larger the period, the smoother the envelope; meanwhile, the interference effect is reduced.

(2) Starting from the UWB pulse signal and GPS signal system, the relationship between the PSD of UWB pulse signal under different pulse repetition frequencies is emphatically explored: when the integer multiple of the pulse repetition frequency happens to fall in the GPS L1 carrier center frequency, the impact on GPS signal interference reaches the maximum. The greater the pulse repetition frequency, the more the energy of the interference signal entering into the GPS receiver, and the more obvious the interference effect. At this time, the $\mathrm{TH}$ code period has less impact on the signal.

(3) We analyzed the equivalent $C / N_{0}$ and demodulation BER of GPS receivers under UWB signal interference 
and established BER parameter models under different UWB signal refrequency. It can be seen that the greater the UWB interference signal power, the more serious the parameter degradation, which has a certain impact on the ranging accuracy of the GPS receiver.

(4) Based on the analysis of the GPS receiver equivalent $C / N_{0}$ under the UWB signal interference, the theoretically calculated lowest interference level of UWB signal is $-130 \mathrm{dBW}$ when the degradation of GPS receiver performance occurs. In the meantime, the equivalent $C / N_{0}$ is $32 \mathrm{~dB} \mathrm{~Hz}$, and the jammingto-signal ratio $\mathrm{J} / \mathrm{S}$ is about $30 \mathrm{~dB}$.

(5) In order to suppress the interference of UWB pulse signals to GPS receivers, the UWB pulse repetition frequency and $\mathrm{TH}$ code period can be adjusted appropriately, and the transmitted signal power should be strictly controlled to minimize the energy of the interference signal falling into the GPS working frequency band.

\section{Data Availability}

No data were used to support this study.

\section{Conflicts of Interest}

The authors declare that there are no conflicts of interest regarding the publication of this paper.

\section{Acknowledgments}

This work was supported by the 13th Five-Year Plan for Equipment Pre-research Project (41409010501).

\section{References}

[1] F. Meng, High-Power Ultra-Wideband Electromagnetic Pulse Technology, National Defense Industry Press, Beijing, China, 2011.

[2] H. Li, Research on Key Technologies of Ultra-Wideband Radar, University of Electronic Science and Technology of China, Chengdu, China, 2000.

[3] J. Chen, Research on Ultra-Wideband Radar Signal Processing and Imaging Methods, Graduate University of Chinese Academy of Sciences (Institute of Electronics), Beijing, China, 2007.

[4] J. R. Foerster, "Interference modeling of pulse-based UWB waveforms on narrowband systems," in Prceedings of the Vehicular Technology Conference IEEE 55th Vehicular Technology Conference VTC Spring 2002 (Cat No02CH37367), Birmingham, AL, USA, May 2002.

[5] K. Y. Sakharov, A. V. Sukhov, V. L. Ugolev, and Y. M. Gurevich, "Study of UWB electromagnetic pulse impact on commercial unmanned aerial vehicle," in Proceedings of the 2018 International Symposium on Electromagnetic Compatibility (EMC EUROPE), Amsterdam, Netherlands, August 2018.

[6] X. Jin, Y. Kang, and B. Li, "The potential interference of ultrawideband pulses to the global positioning system," Journal of Shenyang Ligong University, vol. 4, no. 3, pp. 65-68, 2005.
[7] B. Huang and S. Xiao, "Research on the interference of ultrawideband communication signals to GPS receivers," Journal of Information Engineering University, vol. 4, no. 3, pp. 237240, 2006.

[8] M. K. Bek, E. M. Shaheen, and S. A. Elgamel, "Mathematical analyses of pulse interference signal on post-correlation carrier-to-noise ratio for the global positioning system receivers," IET Radar, Sonar \& Navigation, vol. 9, no. 3, pp. 266-275, 2015.

[9] Z. Zhang, X. Liu, C. Jin et al., "Interference of high-repetition frequency ultra-wide-spectrum short electromagnetic pulses to GPS receivers," High Power Laser and Particle Beam, vol. 26, no. 3, pp. 177-182, 2014.

[10] T. Lu, H. Zhang, X. Cui, and T. A. Gulliver, "Power spectrum analysis of interference to a beidou satellite receiver from UWB impulse radio sysems," in Proceedings of the 2014 12th International Conference on Signal Processing (ICSP), Hangzhou, China, October 2014.

[11] M. Luo, D. Akos, M. Koenig et al., "Testing and research on interference to GPS from UWB transmitters," in Proceedings of the 14th International Technical Meeting of the Satellite Division of The Institute of Navigation (ION GPS 2001), pp. 1-13, Salt Lake City, UT, USA, September 2001.

[12] G. Xie, GPS Principle and Receiver Design, Publishing House of Electronics Industry, Beijing, China, 2017.

[13] Q. Zhang, E. Cheng, Y. Wang et al., "Research on electromagnetic interference effect law of UAV satellite navigation system," Systems Engineering and Electronic Technology, vol. 42, no. 12, pp. 2684-2691, 2020. 